\title{
Discoid Lupus Erythematosus: A Cross-Sectional Study From the Sindh Institute of Skin Diseases, Karachi, Pakistan
}

\author{
Erum Ashraf ${ }^{1}$, Afza N. Ghouse ${ }^{2}$, Sitwat Siddiqui ${ }^{3}$, Sana Siddiqui ${ }^{4}$, Zara Khan ${ }^{5}$ \\ 1. Dermatology, Institute of Skin Diseases Sindh, Karachi, PAK 2. Dermatology, Patel Hospital, Karachi, PAK 3. \\ Dermatology, Dr. Ziauddin Hospital, Karachi, PAK 4. Dermatology, Memon Medical Institute Hospital, Karachi, PAK 5. \\ Dermatology, Medical Glamor Clinics, Riyadh, SAU
}

Corresponding author: Erum Ashraf, drerum_ashraf@yahoo.com

\begin{abstract}
Introduction

Discoid lupus erythematosus (DLE) is the most common form of cutaneous lupus erythematosus. It is a chronic, scar-forming, photosensitive autoimmune dermatosis presenting with erythematous and scaly lesions. Predisposed areas include sun-exposed areas like the nose, forehead, and cheeks, as well as the upper body and extremities. The histological findings are typical, with interface dermatitis. Immunoglobulin M (IgM) and immunoglobulin G (IgG) are the most common deposits in the dermoepidermal junction of the involved skin. The most common treatments used are sunscreens, topical corticosteroids, and antimalarials. Immunosuppressive agents, thalidomide, dapsone, and retinoids can be used in refractory cases. The aim of this study was to study the clinicopathologic patterns of DLE in patients presenting to the Institute of Skin Diseases in Sindh, Karachi.
\end{abstract}

Methods

A total of 53 consecutive patients with DLE meeting the inclusion criteria were evaluated between February 18, 2018 to March 2, 2019 at the Institute of Skin Diseases. Patients with clinical suspicion of DLE were evaluated and studied prospectively after written informed consent was obtained. Information was then collected from their medical histories, physical examination records, and laboratory investigation reports.

Results

A total of 53 consecutive patients with clinical and/or histological diagnosis of DLE was included in this study, out of which $75.5 \%$ (40) were females with a male to female ratio of $1: 3.1$. The mean age of the patients at the time of presentation was $36.02 \pm 10.04$ years, ranging from 14 to 65 years. More than half of the patients $(35,66.0 \%)$ were under 40 years of age and $20.8 \%$ (11) had a positive family history of DLE. DLE was localized in 36 patients (67.9\%) and exposure to the ultraviolet radiation (UVR) was found to be the most frequent induced factor in 46 patients (86.8\%), followed by stress which was observed in 14 patients $(26.4 \%)$. The distribution of commonly affected sites were the face (81.1\%), the limbs (71.7\%), and the scalp (48.4\%) of the patients. Serology antinuclear antibody (ANA) was positive in $56.6 \%$ and serology anti-doublestranded deoxyribonucleic acid antibodies (anti-dsDNA) were positive in $45.3 \%$ of patients. Smoking, as an induced factor, was more commonly observed among male patients as compared to the female patients with a proportion of $53.8 \%$ vs. $2.5 \%$, p $<0.001$, while stress was more common among female patients with a proportion of $35 \%$ vs. $0 \%, p=0.013$, respectively. Histopathology with direct immunofluorescence was done in 33 cases which included cases with negative serology or where the diagnosis was in doubt clinically. The main histopathological features observed were periadnexal and perivascular dermal infiltrates, basal cells vacuolization, epidermal atrophy, hyperkeratosis, and follicular plugging. The commonest morphological form observed was the classic discoid plaque form.

Conclusion

Clinical patterns of DLE in our population comprises of female dominance. Exposure to UVR was the leading inducing factor. The face and limbs were the most commonly involved sites, and the majority of the patients had localized DLE with positive ANA in more than half of those patients. The importance of limiting ultraviolet radiation exposure and toxins (drugs and smoking) should be emphasized in our population.

Categories: Dermatology

Keywords: discoid lupus erythematosus (dle), lupus profundus, tumid lupus, chilblain lupus, lichen planus

\section{Introduction}


Discoid lupus erythematosus (DLE) is a benign inflammatory disorder that is autoimmune in origin. The disease involves mainly the sun-exposed areas of the body, especially the face and scalp, with variable clinical presentations and is characterized by scaly, well-defined red plaques healing with scarring and pigmentary changes [1]. It is approximately three times more common in females as compared to males and most of these females are 40 - 50 years of age [2]. The disease is broadly categorized into a localized variant involving mainly the face and scalp areas and a disseminated form which is widespread extending below the neck area [3]. Other variants include chilblain lupus which is characterized by red to violaceous lesions on the acral parts of the body induced by cold, leading to ulceration and scarring, and lupus profundus characterized by painful subcutaneous nodules and plaques followed by atrophy of fat [4-5]. Other less common forms are hypertrophic or verrucous forms, discoid lupus erythematosus, and lichen planus overlap form, tumid form, linear form, etc. The diagnosis of DLE is usually made clinically. For confirmation of diagnosis, serological and histopathological analysis with immunofluorescence are done. Common serological tests include an antinuclear antibody test (ANA) which is positive in $20 \%$ to $30 \%$ cases (when tested on human cell lines) and anti-double-stranded DNA antibodies (anti-dsDNA) which is positive in 5\% to $20 \%$ cases. Other serological tests, including precipitating anti-Sjögren's-syndrome-related antigen A (Ro/SSA) and anti-Sjögren's-syndrome-related antigen B (La/SSB) autoantibodies, are rare in DLE [6]. However, low levels of anti-Ro/SSA antibodies detected by enzyme-linked immunoassays are more common [6]. The histopathologic features of DLE include epidermal atrophy, hyperkeratosis, follicular plugging, vacuolar degeneration of basal cells, thickening of the basement membrane, perivascular, periappendiceal lymphocytic cell infiltrate, and sometimes mucin deposition [7]. For further confirmation, we looked for the deposition of immunoreactants by direct immunofluorescence at the dermoepidermal junction. These immunoreactants, which include immunoglobulins with or without complements, are usually positive in the granular pattern at the dermoepidermal junction of lesional skin in around 90\% of cases [8-9].

There are scanty studies on DLE in the Pakistani population which is said to be the commonest variant of cutaneous lupus erythematosus worldwide. Knowledge of its clinical spectrum, epidemiology, and diagnostic tests are important for a timely diagnosis and hence early treatment of the disease since a delay in diagnosis is associated with complications and poor emotional impact.

\section{Materials And Methods}

This was a cross-sectional study with a total of 53 consecutive patients of both genders, aged between 14 to 65 years, who were admitted with clinical suspicion of discoid lupus erythematosus in both inpatient and outpatient units of the Institute of Skin Diseases, Sindh, Karachi. The sample size was calculated using the World Health Organization (WHO) software, version 2.0. The study by Bajaj et al. was taken as a reference study for sample size calculation, in which the face was found to be the commonest site, accounting for $54.5 \%$ [10]. As our sample size was small, we had to set the margin of error by $14 \%$ rather than the standard $10 \%$ and confidence interval as $94 \%$ to calculate the sample size. The sample size for our study came out to be 47 patients which, after adjusting $10 \%$ as lost to follow-up patients, a final total of 53 patients were recruited during the study period. These patients were evaluated between February 18, 2018 to March 2, 2019 at the Institute of Skin Diseases and studied prospectively. Patients with systemic manifestations of the disease and mixed connective tissue disorders were excluded from the study. Those patients who were younger than 14 and older than 65 years of age, pregnant patients, and those not willing to give consent were also excluded. Written informed consent was obtained from all patients.

The medical records of all patients were obtained in detail according to the proforma which included age, gender, family history, distribution of the lesions of DLE, and type of DLE. Induced factors like ultraviolet light, viral infections, drugs, smoking, and trauma were also recorded. The distinction between localized and disseminated lesions were solely made on cutaneous involvement; lesions limited to the head and neck were labeled as the localized variety and lesions beyond the neck were labeled as the disseminated variety. The routine laboratory serology blood tests (ANA, dsDNA, anti-Ro SSA, anti-La SSB, and anti-Sm) were analyzed by standard methods. Serum ANA was tested preferably by human epithelial type 2 (HEp-2) cells using immunofluorescence technique or by enzyme-linked immunosorbent assay (ELISA) method. Anti-dsDNA antibody levels were analyzed by radioimmunoassay (RIA) or ELISA. Wherever needed, histopathology analysis was done and recorded on the proforma as suggestive or not suggestive of DLE. Tissues for histological examination were fixed in $10 \%$ buffered formalin and processed for hematoxylin and eosin stain. Histopathology findings were further confirmed on the detection of immunoreactants at the dermoepidermal junction by the direct immunofluorescence technique in which the biopsy samples were placed in Michel's medium and stored at $4^{\circ} \mathrm{C}$ until further processed. In the statistical analysis, the descriptive variables were presented as frequency, percentage, mean or standard deviation, and the results were reported. For comparison of gender and groups among the different characteristics of the patients, the chi-squared test/Fisher exact test were analyzed by using the Statistical Package of Social Sciences (SPSS), version 21 (IBM SPSS Statistics, Armonk, NY).

\section{Results}

A total of 53 consecutive patients with DLE was included in this study, out of which $75.5 \%(n=40)$ were females with a male to female ratio of $1: 3.1$. The mean age of the patients at the time of presentation was $36.02 \pm 10.04$ years (ranging from 14 to 65 years). More than half of the patients $(n=35,66.0 \%)$ were under 


\section{Cureus}

40 years of age and 11 (20.8\%) had a positive family history. DLE was localized in $67.9 \%(n=36)$ of the patients and exposure to the ultraviolet radiation (UVR) was found to be the most frequent induced factor, $86.8 \%(n=46)$, followed by stress which was observed in $26.4 \%(n=14)$ of the patients. Among multiple response categories in the face, the cheek was found to be the commonest site involved in $76.7 \%(n=33)$ cases, followed by the nose $60.5 \%$, forehead $48.8 \%$, eyelid $39.5 \%$, and trunk $37.2 \%$ cases, respectively.

The distribution of commonly affected sites are the face in $81.1 \%$, limbs in $71.7 \%$, and scalp in $48.4 \%$ of the patients. Serology antinuclear antibody (ANA) was positive in $56.6 \%$ and serology anti-dsDNA was positive in $45.3 \%$. Clinical patterns of discoid lupus erythematosus by gender are presented in Table 1 . 


\section{Cureus}

\begin{tabular}{|c|c|c|c|c|}
\hline \multirow{2}{*}{ Characteristics } & \multirow{2}{*}{ Total } & \multicolumn{2}{|l|}{ Gender } & \multirow{2}{*}{ P-value } \\
\hline & & Male & Female & \\
\hline Sample size (N) & 53 & 13 & 40 & - \\
\hline \multicolumn{5}{|l|}{ INDUCED FACTORS } \\
\hline Ultraviolet radiation (UVR) & $86.8 \%(46)$ & $76.9 \%(10)$ & $90 \%(36)$ & 0.226 \\
\hline Viral infection ${ }^{1}$ & $13.2 \%(7)$ & $7.7 \%(1)$ & $15 \%(6)$ & 0.499 \\
\hline Drugs $^{2}$ & $9.4 \%(5)$ & $0 \%(0)$ & $12.5 \%(5)$ & 0.18 \\
\hline Smoking & $15.1 \%(8)$ & $53.8 \%(7)$ & $2.5 \%(1)$ & $<0.001^{*}$ \\
\hline Trauma & $9.4 \%(5)$ & $7.7 \%$ (1) & $10 \%(4)$ & 0.805 \\
\hline Stress & $26.4 \%(14)$ & $0 \%(0)$ & $35 \%$ (14) & $0.013^{\star}$ \\
\hline \multicolumn{5}{|l|}{ TYPES } \\
\hline Localized & $67.9 \%(36)$ & $38.5 \%(5)$ & $77.5 \%(31)$ & \multirow{2}{*}{$0.009^{*}$} \\
\hline Disseminated & $32.1 \%(17)$ & $61.5 \%$ (8) & $22.5 \%(9)$ & \\
\hline \multicolumn{5}{|l|}{ SITE OF INVOLVEMENT } \\
\hline Scalp & $48.4 \%(16)$ & $50 \%(3)$ & $48.1 \%(13)$ & 0.52 \\
\hline Face & $81.1 \%(43)$ & $92.3 \%$ (12) & $77.5 \%$ (31) & 0.236 \\
\hline Cheeks & $76.7 \%$ (33) & 83.3\% (10) & $74.2 \%(23)$ & 0.209 \\
\hline Nose & $60.5 \%(26)$ & $66.7 \%(8)$ & $58.1 \%(18)$ & 0.300 \\
\hline Forehead & $48.8 \%(21)$ & $41.7 \%(5)$ & $51.6 \%(16)$ & 0.922 \\
\hline Ears & $39.5 \%(17)$ & $33.3 \%(4)$ & $41.9 \%(13)$ & 0.908 \\
\hline Trunk & $37.2 \%(16)$ & $33.3 \%(4)$ & $38.7 \%(12)$ & 0.958 \\
\hline Limbs & $71.7 \%$ (38) & $69.2 \%$ (9) & $72.5 \%(29)$ & 0.82 \\
\hline Upper limbs & $57.9 \%$ (22) & $22.2 \%(2)$ & $69 \%(20)$ & \multirow{2}{*}{$0.013^{*}$} \\
\hline Lower limbs & $42.1 \%(16)$ & $77.8 \%(7)$ & $31 \%(9)$ & \\
\hline \multicolumn{5}{|l|}{ SEROLOGY ANA } \\
\hline Positive & $56.6 \%(30)$ & $30.8 \%(4)$ & $65 \%(26)$ & \multirow{2}{*}{$0.031^{*}$} \\
\hline Negative & $43.4 \%(23)$ & $69.2 \%$ (9) & $35 \%(14)$ & \\
\hline \multicolumn{5}{|l|}{ SEROLOGY DNA } \\
\hline Positive & $45.3 \%(24)$ & $38.5 \%$ (5) & $47.5 \%(19)$ & \multirow{2}{*}{0.57} \\
\hline Negative & $54.7 \%(29)$ & $61.5 \%$ (8) & $52.5 \%(21)$ & \\
\hline
\end{tabular}

\section{TABLE 1: Clinical Patterns of Discoid Lupus Erythematosus by Gender}

* Significant at $5 \%$

1 Viral infections (herpes zoster virus, old smallpox vaccination)

${ }^{2}$ Drugs (isoniazid, penicillamine. griseofulvin, dapsone, hydralazine)

ANA: antinuclear antibody; DNA: deoxyribonucleic acid

Smoking as an induced factor was more commonly observed among male patients as compared to the female patients with a proportion of $53.8 \%$ versus $2.5 \%(p<0.001)$, while stress was more common 


\section{Cureus}

among female patients with a proportion of $35 \%$ vs. $0 \%(p=0.013)$. DLE was localized in more female patients than males with rates of $77.5 \%$ vs. $38.5 \%(p=0.009)$; similarly, positive serology ANA was found to be associated with female gender with rates of $65 \%$ vs. $30.8 \%(p=0.031)$. However, no major differences in the clinical patterns of DLE were observed for the different age groups of the patients. The clinical patterns of discoid lupus erythematosus by age are presented in Table 2. 


\section{Cureus}

\begin{tabular}{|c|c|c|c|}
\hline Characteristics & Age Group (\% / n) 14 to 40 years & Age Group (\% / n) 41 to 65 years & P-value \\
\hline Sample size (N) & 35 & 18 & - \\
\hline \multicolumn{4}{|l|}{ INDUCED FACTORS } \\
\hline Ultraviolet radiation (UVR) & $88.6 \%(31)$ & $83.3 \%(15)$ & 0.594 \\
\hline Viral infection ${ }^{1}$ & $11.4 \%(4)$ & $16.7 \%(3)$ & 0.594 \\
\hline Drugs $^{2}$ & $8.6 \%(3)$ & $11.1 \%(2)$ & 0.765 \\
\hline Smoking & $11.4 \%(4)$ & $22.2 \%(4)$ & 0.299 \\
\hline Irauma & $5.1 \%(2)$ & $16.1 \%(3)$ & 0.196 \\
\hline Stress & $31.4 \%(11)$ & $16.7 \%(3)$ & 0.248 \\
\hline \multicolumn{4}{|l|}{ TYPES } \\
\hline Localized & $74.3 \%(26)$ & $55.6 \%(10)$ & \multirow{2}{*}{0.167} \\
\hline Disseminated & $25.7 \%(9)$ & $44.4 \%(8)$ & \\
\hline \multicolumn{4}{|l|}{ SIIE OF INVOLVEMENI } \\
\hline Scalp & $42.8 \%(9)$ & $58.3 \%(7)$ & 0.322 \\
\hline Face & $82.9 \%(29)$ & $77.8 \%(14)$ & 0.654 \\
\hline Cheeks & $72.4 \%(21)$ & $85.1 \%$ (12) & 0.635 \\
\hline Nose & $55.2 \%(16)$ & $71.4 \%$ (10) & 0.497 \\
\hline Forehead & $41.4 \%(12)$ & $64.3 \%(9)$ & 0.268 \\
\hline Ears & $27.6 \%(8)$ & $64.3 \%(9)$ & $0.045^{\star}$ \\
\hline Irunk & $37.9 \%(11)$ & $35.1 \%$ (5) & 0.184 \\
\hline Limbs & $74.3 \%(26)$ & $66.7 \%(12)$ & 0.560 \\
\hline Upper limbs & $53.8 \%(14)$ & $66.1 \%(8)$ & \multirow{2}{*}{0.457} \\
\hline Lower limbs & $46.2 \%$ (12) & $33.3 \%(4)$ & \\
\hline \multicolumn{4}{|l|}{ SEROLOGY ANA } \\
\hline Positive & $60 \%(21)$ & $50 \%(9)$ & \multirow{2}{*}{0.487} \\
\hline Negative & $40 \%(14)$ & $50 \%(9)$ & \\
\hline \multicolumn{4}{|l|}{ SEROLOGY DNA } \\
\hline Positive & $45.7 \%(16)$ & $44.4 \%(8)$ & \multirow{2}{*}{0.93} \\
\hline Negative & $54.3 \%$ (19) & $55.6 \%(10)$ & \\
\hline
\end{tabular}

\section{TABLE 2: Clinical Patterns of Discoid Lupus Erythematosus by Age}

* Significant at $5 \%$

${ }^{1}$ Viral infection (herpes zoster, old smallpox vaccination)

${ }^{2}$ Drugs (isoniazid, penicillamine, griseofulvin, dapsone, hydralazine)

ANA: antinuclear antibody; DNA: deoxyribonucleic acid

Histopathology with direct immunofluorescence was done in 33 cases where the diagnosis was in doubt. The main histopathological features observed were periadnexal and perivascular dermal infiltrates (32/33), basal cells vacuolization (30/33), epidermal atrophy (29/33), hyperkeratosis (29/33), and follicular plugging in 
Regarding the morphological variants, a classic discoid plaque was present in 36/53 (68\%) patients, followed by the hypertrophic form in $7 / 53$ (13\%). Discoid lupus erythematosus with lichen planus overlap and lupus profundus forms each were present in 4/53 (7.54\%) patients. Linear DLE was diagnosed in 2/53 (4.3\%) cases.

\section{Discussion}

Discoid lupus erythematosus (DLE) is the most common type of chronic cutaneous lupus erythematosus. It can present with different clinical patterns. Hence, in this study, we sought to present clinicopathologic patterns of DLE in the Pakistani population [10]. A higher female to male ratio of 3.1:1 among DLE patients is a common finding in this population. Female patients outnumbered male patients not only in our population but it is also a common observation for various other geographies. The most common age group affected was 14 - 40 years of age with a mean age of onset at 29.4 years which is comparable to other studies [10-13]

Along with the various factors leading to DLE, such as exposure to ultraviolet radiation (UVR), toxins (drugs and smoking), and genetic predisposition, it is believed that hormonal factors, such as estrogen, play a causative role and increases the risk of DLE among females [14]. Stress was also a predominant factor associated with disease activity in $80 \%$ of cases, an important factor which was also proved by a Western study by Chen et al. [15]. Smoking also showed a strong association with DLE in our study, a factor already proved important as an inducing factor by other studies [16]. Six of our patients developed DLE after intake of anti-tuberculous treatment which is a known drug to induce DLE as proved by other studies, while one patient developed the disease after the intake of hydralazine [17-18].

Occupational or non-occupational exposure to UVR is the most common induced factor of DLE in our population, followed by stress, smoking, and viral infection. These observations were in concordance with the literature [12, 19-20]. A major portion of our population experiences an average longer duration of exposure to UVR due to their affiliation with outdoor occupations, such as labor, farming, small street businesses, and working for daily wages [10]. The release of pro-inflammatory cytokines during the apoptosis of keratinocytes due to UV light induces inflammatory changes resulting in DLE [19]. Along with other environmental factors, smoking is considered to play a significant role in the pathogenesis of autoimmune diseases. It was also found to be associated with DLE development [13, 21].

DLE was observed to be localized in the majority of the patients which was in concordance with the results observed in the past studies [10-13]. Disseminated DLE was more common in male patients as compared to females. Post-inflammatory pigmentation is frequently seen in skin types III to V, which are the commonest skin types in our population, contributing to residual pigmentation of lesions. In our study, hydroxychloroquine and azathioprine drugs were commonly used for the treatment of DLE which was also reported by Wahie et al. [13]. ANA serology was positive in a significant number of patients (56.6\%) and was preferably requested on HEp-2 cells to decrease the chances of false-negative results. These results were in contrast to the observations of Bajaj et al. [10] who reported only 17.3\% cases with positive ANA serology. A similar study by Gopalan et al. reported $73 \%$ of cases with positive ANA serology and a strong association with the female gender [12].

Patients with autoantibodies to ds-DNA and anti-Ro were thoroughly checked for SLE; fortunately, none of these patients had clinical and laboratory evidence of SLE. We compared our results with a large multicentre Western study conducted by Biazar et al. [22], a local study conducted by Bajaj et al. [10], and a literature review done in Saudia Arabia by AlSaif [23] and observed that our results were comparable.

The most common site of lesion was the face, which was also observed in $81.1 \%$ of patients in our study. The cheeks, nose, and forehead were the common lesional sites on the face [10, 12]. Limb involvement was surprisingly high in our study (71.7\%). The scalp was found to be involved in $37.7 \%$ of the patients which was in concordance with the range of frequency, $23.6 \%$ to $41 \%$, reported in past studies [24-25].

Histopathology with direct immunofluorescence was done in 33 cases which included cases with negative serology or where the diagnosis was in doubt clinically. The main histopathological features observed were periadnexal and perivascular dermal infiltrates 32/33, basal cell vacuolization 30/33, epidermal atrophy $29 / 33$, hyperkeratosis $29 / 33$, and follicular plugging in $27 / 33$ cases. These are common findings observed in the histopathological examination as mentioned in the literature and are important clues to confirm the diagnosis [25-26].

Regarding the morphological variants, the classic discoid plaque was present in $36 / 53$ (68\%), followed by the hypertrophic form in 7/53 (13\%) of patients. Discoid lupus erythematosus with lichen planus overlap form and lupus profundus forms each were present in 4/53 (7.54\%) of patients. Linear DLE was diagnosed in 2/53 (4.3\%) cases. It is said to be a unique and one of the rarest forms of DLE with only a few reported cases in the literature [27-28]. 
DLE is a disease characterized by inflammatory plaques. Currently, very limited data are available on the effectiveness of available treatment options. No medication has been approved specifically for DLE, although many drugs have been used for the management and treatment of DLE [29-30]. A systematic review of clinical trials summarized the available evidence and concluded that fluocinonide creams can be more effective for DLE skin lesions as compared to hydrocortisone [30]. For complete resolution, hydroxychloroquine and azathioprine were found to have equal efficacy. However, acitretin therapy had a more adverse event rate than hydroxychloroquine. Quality randomized control trials are needed to deduce any conclusion with certainty regarding the treatment of DLE [30].

The findings of this study have to be seen in the light of a few limitations. There are three major limitations in this study that could be addressed in future research. The first is that our study is a single centre experience, the second limitation concerns time constraints and a small number of patients, and the third one relates to limited previous studies locally.

\section{Conclusions}

Clinicopathologic patterns of DLE in our population comprises of female dominance, the face and limbs were the most common sites, exposure to the ultraviolet radiation (UVR) was the leading inducing factor, and the majority of the patients had localized DLE with positive ANA. The importance of limiting ultraviolet radiation exposure and the use of toxins (drugs and smoking) should be emphasized in our population. Similarly, patients with disseminated DLE should be regularly followed as these cases carry a risk of transmission into squamous cell carcinoma. Hence, we conclude that it is very important for healthcare providers, especially dermatologists, to have adequate knowledge about the signs and symptoms of DLE and its variants to ensure early detection and treatment.

\section{Additional Information}

\section{Disclosures}

Human subjects: Consent was obtained by all participants in this study. Institutional Review Board, Institute of Skin Diseases, Sindh, Karachi issued approval 1453. Animal subjects: All authors have confirmed that this study did not involve animal subjects or tissue. Conflicts of interest: In compliance with the ICMJE uniform disclosure form, all authors declare the following: Payment/services info: All authors have declared that no financial support was received from any organization for the submitted work. Financial relationships: All authors have declared that they have no financial relationships at present or within the previous three years with any organizations that might have an interest in the submitted work. Other relationships: All authors have declared that there are no other relationships or activities that could appear to have influenced the submitted work.

\section{References}

1. McDaniel B, Sukumaran S, Tanner LS: Discoid Lupus Erythematosus. StatPearls [Internet]. StatPearls Publishing, Treasure Island (FL); 2020.

2. Jarukitsopa S, Hoganson DD, Crowson CS, et al.: Epidemiology of systemic lupus erythematosus and cutaneous lupus erythematosus in a predominantly white population in the United States. Arthritis Care Res (Hoboken). 2015, 67:817-828.

3. Kuhn A, Sticherling M, Bonsmann G: Clinical manifestations of cutaneous lupus erythematosus. J Dtsch Dermatol Ges. 2007, 5:1124-1137.

4. Hedrich C, Fiebig B, Hauck F, et al.: Chilblain lupus erythematosus-a review of literature . Clin Rheumatol. 2008, $27: 949-954.10 .1007 / \mathrm{s} 10067-008-0942-9$

5. Fraga J, García-Díez A: Lupus erythematosus panniculitis. Dermatol Clin. 2008, 26:453-463. 10.1016/j.det.2008.06.002

6. Cozzani E, Drosera M, Gasparini G, Parodi A: Serology of lupus erythematosus: correlation between immunopathological features and clinical aspects. Autoimmune Dis. 2014, 2014:321359. $10.1155 / 2014 / 321359$

7. Grönhagen C, Fored C, Granath F, Nyberg F: Cutaneous lupus erythematosus and the association with systemic lupus erythematosus: a population-based cohort of 1088 patients in Sweden. Br J Dermatol. 2011, 164:1335-1341. 10.1111/j.1365-2133.2011.10272.x

8. Bharti S, Dogra S, Saikia B, Walker RM, Chhabra S, Saikia UN: Immunofluorescence profile of discoid lupus erythematosus. Indian J Pathol Microbiol. 2015, 58:479-482. 10.4103/0377-4929.168850

9. Naqqash S, Asad F, Pal SS: Direct immunofluorescence and histopathology in chronic discoid lupus erythematosus. J Pak Assoc Dermatol. 2011, 21:98-101.

10. Bajaj DR, Devrajani BR, Matlani BL: Discoid lupus erythematosus: a profile. J Coll Physicians Surg Pak. 2010, 20:361-364.

11. Böckle BC, Sepp NT: Smoking is highly associated with discoid lupus erythematosus and lupus erythematosus tumidus: analysis of 405 patients. Lupus. 2015, 24:669-674. 10.1177/0961203314559630

12. Gopalan G, Gopinath SR, Kothandaramasamy R, Pandian S: A clinical and epidemiological study on discoid lupus erythematosus. Int J Res Dermatol. 2018, 4:396-402. 10.18203/issn.24554529.IntJResDermatol20183165

13. Wahie S, Daly AK, Cordell HJ, et al.: Clinical and pharmacogenetic influences on response to hydroxychloroquine in discoid lupus erythematosus: a retrospective cohort study. J Invest Dermatol. 2011, 131:1981-1986. 10.1038/jid.2011.167 
14. Askanase AD: Estrogen therapy in systemic lupus erythematosus . Treat Endocrinol. 2004, 3:19-26. 10.2165/00024677-200403010-00003

15. Chen P, Broadbent E, Coomarasamy C, Jarrett P: Illness perception in association with psychological functioning in patients with discoid lupus erythematosus. Br J Dermatol. 2015, 173:824-826. 10.1111/bjd.13709

16. Chasset F, Francès C, Barete S, Amoura Z, Arnaud L: Influence of smoking on the efficacy of antimalarials in cutaneous lupus: a meta-analysis of the literature. J Am Acad Dermatol. 2015, 72:634-639. 10.1016/j.jaad.2014.12.025

17. Vedove CD, Del Giglio M, Schena D, Girolomoni G: Drug-induced lupus erythematosus. Arch Dermatol Res. 2009, 301:99-105. 10.1007/s00403-008-0895-5

18. Richardson BC: Drug-induced lupus erythematosus. Dubois' Lupus Erythematosus and Related Syndromes, 9th edition. Wallace DJ, Hahn BH (ed): Elsevier, New York; 2019. 377-388.

19. Kim A, Chong BF: Photosensitivity in cutaneous lupus erythematosus. Photodermatol Photoimmunol Photomed. 2013, 29:4-11. 10.1111/phpp.12018

20. Szczęch J, Samotij D, Werth VP, Reich A: Trigger factors of cutaneous lupus erythematosus: a review of current literature. Lupus. 2017, 26:791-807. 10.1177/0961203317691369

21. Miot HA, Bartoli Miot LD, Haddad GR: Association between discoid lupus erythematosus and cigarette smoking. Dermatology. 2005, 211:118-122. 10.1159/000086440

22. Biazar C, Sigges J, Patsinakidis N, et al.: Cutaneous lupus erythematosus: first multicenter database analysis of 1002 patients from the European Society of Cutaneous Lupus Erythematosus (EUSCLE). Autoimmun Rev. 2013, 12:444-454. 10.1016/j.autrev.2012.08.019

23. AlSaif F: Discoid lupus erythematosus in Saudi Arabia: a literature review . Lupus Open Access. 2016, 1:1-3. 10.35248/2684-1630.16.1.105

24. Al-Hattab MK, Al-Waiz M: Discoid lupus erythematosus in Iraqi patients: a clinical and histopathological study. Ann Saudi Med. 2004, 24:289-292. 10.5144/0256-4947.2004.289

25. Al-Saif FM, Al-Balbeesi AO, Al-Samary AI, Al-Rashid SB, Halwani M, Al-Mekhadab E, Al-Kelabi M: Discoid lupus erythematosus in a Saudi population: clinical and histopathological study. J Saudi Soc Dermatol Dermatol Surg. 2012, 16:9-12. 10.1016/j.jssdds.2011.09.003

26. Jarrett P, Werth VP: A review of cutaneous lupus erythematosus: improving outcomes with a multidisciplinary approach. J Multidiscip Healthc. 2019, 12:419-428. 10.2147/JMDH.S179623

27. Alcántara-González J, Fernandez-Guarino M, Carrillo-Gijon R, Jaén-Olasolo P: Linear cutaneous lupus erythematosus. Indian J Dermatol Venereol Leprol. 2011, 77:717-719.

28. Kim J, Lee SH, Roh MR: Linear cutaneous lupus erythematosus on the midline of the face . J Dermatol. 2011, 38:609-612. 10.1111/j.1346-8138.2010.01013.x

29. Garza-Mayers AC, McClurkin M, Smith GP: Review of treatment for discoid lupus erythematosus . Dermatol Ther. 2016, 29:274-283. 10.1111/dth.12358

30. Jessop S, Whitelaw DA, Grainge MJ, Jayasekera P: Drugs for discoid lupus erythematosus. Cochrane Database Syst Rev. 2017, 5:CD002954. 10.1002/14651858.CD002954.pub3 